\title{
Social Responses to Media Technologies in the 21 st Century: The Media Are Social Actors Paradigm
}

\author{
Matthew Lombard ${ }^{1}$ and Kun $\mathrm{Xu}^{2}$ (1) \\ 1 Klein College of Media and Communication, Temple University, Philadelphia, PA, USA \\ 2 College of Journalism and Communications, University of Florida, Gainesville, FL, USA
}

\begin{abstract}
Clifford Nass and his colleagues proposed the Computers Are Social Actors (CASA) paradigm in the 1990s and demonstrated that we treat computers in some of the ways we treat humans. To account for technological advances and to refine explanations for CASA results, this paper proposes the Media Are Social Actors (MASA) paradigm. We begin by distinguishing the roles of primary and secondary cues in evoking medium-as-social-actor presence and social responses. We then discuss the roles of individual differences and contextual factors in these responses and identify mindless and mindful anthropomorphism as two major complementary mechanisms for understanding MASA phenomena. Based on evolutionary psychology explanations for socialness, we conclude with nine formal propositions and suggestions for future research to test and apply MASA.
\end{abstract}

Keywords: computers are social actors, media are social actors, medium-as-social-actor presence, social presence, social cues, mindlessness, anthropomorphism

\section{Introduction}

Among the more surprising research results in the study of human-computer interaction are those of Clifford Nass and his colleagues demonstrating that computer users apply social rules from human-human interactions when they use computers. A series of experiments demonstrated that users follow social rules based on gender, team membership, politeness,

CONTACT Kun Xu (D) - College of Journalism and Communications • University of Florida • 1885 Stadium Rd, Gainesville,

FL 32611 • kun.xu@ufl.edu 
and more (Nass et al., 1994; Nass et al., 1996; Nass et al., 1997). Nass proposed the Computers are Social Actors (CASA) paradigm to describe the phenomenon and an approach to studying it and expanded on the idea with Byron Reeves in their book The Media Equation (Nass et al., 1994; Reeves \& Nass, 1996).

The original CASA paradigm was proposed over a quarter century ago. Today we are surrounded by many more media technologies, including wearable devices, smartphones, digital assistants, and humanoid robots (Carolus et al., 2019; Kanda et al., 2009; Perry, 2014). While growing research has been applying the CASA paradigm to understand interactions with these emerging technologies, the general tenet that users respond to computers and other media technologies as if they were real people requires more subtle and detailed explications that account for media users' behavior. Although the CASA paradigm and the Media Equation describe a series of experimental findings about individuals' social reactions to technologies, one caveat to the theoretical framework is that it lacks explicit propositions that researchers can examine, test, and refine. Thus, to build a more refined framework, we propose the Media are Social Actors (MASA) paradigm as a structured extension of the CASA paradigm. We suggest that an enhanced framework that builds on the CASA paradigm, expounds the effects of social cues, describes the psychological mechanism of social responses, and provides propositions that scholars can test will not only direct future research on human-technology interaction but also meet the criteria of explanatory power, predictive power, falsifiability, heuristic value, and internal consistency that Chaffee and Berger (1987) outlined for a rigorous theoretical framework.

We expand the CASA paradigm from the perspective of social cues, as social cues are a key factor that leads to users' social responses. For example, Nass (2004) noted that social cues which may trigger applying etiquette rules to computers include language, voice, a face, emotion manifestation, interactivity, perceived engagement with the user, and filling of social roles. Another reason to approach the expansion of the paradigm through social cues is that cues can evoke medium-as-social-actor presence. Medium-as-social-actor presence refers to the idea that when a medium itself presents social cues, individuals perceive it (with or without conscious awareness) not as a medium but as an independent social entity (Lombard \& Ditton, 1997). While sometimes researchers use social presence to describe medium-as-social-actor presence experiences, social presence may involve other meanings such as the "subjective quality of the medium" (Short et al., 1976, p. 66) or parasocial interaction (Horton \& Wohl, 1956). Thus, in this paper, we will consistently use the term medium-as-social-actor presence to avoid misunderstandings (for definitions of other types of social presence, see Biocca et al., 2003; Lombard \& Jones, 2015). Although not all CASA studies have examined medium-as-social-actor presence, and presence need not always lead to behavioral social responses, social responses can be considered as a reflection and indication of users' medium-as-social-actor presence experiences.

While much literature has examined the effects of social cues on users' social responses to technologies (e.g., Araujo, 2018; Nomura \& Kanda, 2015; Terzioğlu et al., 2020), most research has focused on either the effects of single social cues or the cumulative effects of social cues. A large body of research suggests that technologies with more social cues can evoke stronger social responses than ones with fewer social cues (e.g., Burgoon et al., 2000; Ghazali et al., 2018; Tung \& Deng, 2007). By contrast, limited research has clarified the distinctions among these social cues. It is likely that individual social cues can exert 
different effects on users' responses (Abubshait \& Wiese, 2017). Therefore, in this paper, we suggest that (1) the quality of social cues should receive more attention in future research, (2) the roles of individual differences and communication contexts should be considered in the paradigm, (3) the two prominent psychological explanations for social responses, mindlessness and anthropomorphism, can be unified and applied to explain a wide variety of human-machine communication (HMC) scenarios, and (4) specific propositions should be developed and refined to derive future research questions on HMC.

Expanding the CASA paradigm to the MASA paradigm through the examination of social cues has three major benefits. First, from a theory construction perspective, broadening the CASA paradigm will expand the value and applicability of an important theoretical framework. By refining and clarifying the concepts, assumptions, and propositions of the MASA paradigm, researchers can better understand, explain, predict the effects of, and generate new questions about our increasingly common communication experiences with diverse technologies.

Second, when interaction with technologies mimics natural human communication, people expend less cognitive effort (Gambino et al., 2020), making the experience more efficient and intuitive. And when interactions account for users' personalities, identities, and demographic characteristics along with the communication context, the experience is further enhanced. We can therefore apply the propositions of the MASA paradigm to improve the design, enhance the users' experience, and therefore increase the success, of a variety of media technologies (Bartneck et al., 2008; Biocca et al., 2003).

Third, expanding CASA to MASA highlights important ethical issues in technology development. As designers can more easily embed different social cues in technologies, we will need to consider the potential for unethical manipulation of user responses. Those who devise, promote, adopt, and study presence-evoking technologies should be mindful of their concomitant risks (Lombard, 2009).

\section{The Roles of Cues, Individual Differences, and Contextual Factors}

To elaborate the mechanism of the MASA paradigm, below we first explicate the role of social cues. We demonstrate the intrinsic distinctions among social cues, the effects of social cues on medium-as-social-actor presence and social responses, and then briefly introduce the role of individual differences and contextual factors in our theoretical framework.

\section{Social Cues}

Although many researchers have studied the effects of social cues (Tanis \& Postmes, 2003; Walther et al., 2005; Wang et al., 2010), few have explicitly defined the term. Social cues can serve as affordances (Sundar et al., 2015), reflect physical attractiveness (Antheunis \& Schouten, 2011), or be conceived as contextual information (Sproull \& Kiesler, 1986). Here we specifically adopt Fiore et al.s (2013) definition of cues as "biologically and physically determined features salient to observers because of their potential as channels of useful information" (p. 2). Fiore and colleagues distinguished social cues and social signals, where social cues can be understood as physical or behavioral features displayed by a social actor 
and social signals are the meaningful interpretations of these social cues by the perceivers. This is an important stepping-off point because social cues have been used interchangeably with social signals in some literature (Wiltshire et al., 2014). Examples of social cues include a social actor's voice, humanlike appearance, and eye gaze, whereas social signals include perceivers' translation of these and other cues into an understanding of the social actor's emotion (e.g., indicated by the actor's smiling face), attention (e.g., indicated by eye contact), empathy (e.g., indicated by hugs or language), and so on.

Based on this distinction, although the early CASA researchers manipulated computers to have personalities, genders, or conversation patterns, what they actually controlled were various sets of social cues. For instance, in their manipulation of gendered computers, Nass et al. (1997) assigned a female voice and a male voice to the computers and found that a female computer was perceived to be more familiar with love and relationships, whereas a male computer was more knowledgeable about technical subjects. Similarly, when operationalizing computer personalities, Nass and Lee (2001) controlled the levels of speech rate, volume, fundamental frequency, and pitch range to differentiate introverted and extroverted computers.

Although the role of social cues has been examined in much of the CASA literature, one question that Nass and Moon (2000) raised in their suggestions for future research is whether there exist some dimensions of a computer that are more likely to evoke social responses compared to others, and how different combinations of them would impose additive or synergetic influence on social responses. Although no sufficient findings have been established to advance a hierarchy of social cues which indicates the distinct power of each single cue over users' presence experiences and social responses, some scholarship at least organizes and compares various pairs of social cues. For example, Nass and Steuer (1993) examined the effects of different voices and of the same or different computer boxes producing the voices. They found that the voice manipulation had greater effects than the box one in predicting perceived accuracy and fairness of an evaluation session in humancomputer interaction. Reeves and Nass (2000) also noted that sight and sound dominate human perception and play a more important role than other senses that can relay social cues like smell. Thus, it can be inferred that there should be a group of cues that are more likely to generate individuals' social perception than others. This postulation is consistent with the cognitive miser theory (Fiske \& Taylor, 1991), based on which researchers suggest that how a person is construed is determined by the quality of available visual inputs. The cognitive process of person perception may occur automatically when critical cues such as facial features are available to the perceiver, but the same process may not be activated without the presence of these cues (Gauthier \& Tarr, 1997; Martin \& Macrae, 2007). Therefore, in the process of activating users' medium-as-social-actor presence and social responses, what matters is not only the quantity of social cues, but also the quality of social cues.

Based on prior research, we identify two groups of social cues, primary cues and secondary cues, to refer to their different effects on users' social reactions. Primary cues are those that are most salient and central to humans' perception of socialness. Responses to these primary cues are based on individuals' evolutionary bias toward humanlike (or animal-like) characteristics. Each primary social cue should be sufficient but not necessary to evoke social responses. By contrast, secondary cues are those that are less salient and less 
central to humans' perception of socialness. They are neither sufficient nor necessary to evoke social responses.

\section{Primary Cues}

We include face, eye gaze, gesture, human-sounding voice, and humanlike or animal-like shape as major examples of primary cues. These cues have been found to evoke humans' evolutionarily based responses. For instance, researchers have found that infants are sensitive to fearful, surprising, and angry facial expressions (Kobiella, et al., 2008; Schmidt \& Cohn, 2001; Serrano et al., 1992). Paredolia, which refers to the illusion in which people see faces in inanimate objects (e.g., taking a car's headlights as eyes and its bumper or grill as mouth) (Takahashi \& Watanabe, 2013), also supports the idea that the face is a powerful cue in triggering social perception. Past research has corroborated that including a face as a social cue can lead participants to perceive computer agents as more trustworthy, persuasive, and positive (Gong, 2008; Shamekhi et al., 2018).

As an important social feature of a face, eye gaze is another evolutionarily significant characteristic of human beings. Eye gaze delivers the social signals of attention, emotion, or acknowledgment (Andrist et al., 2015; Fink \& Penton-Voak, 2002). Past research has shown that even 12-month-old infants can follow the gaze direction of robots (Okumura et al., 2013).

Another social cue that serves as a basic element of our daily nonverbal interaction is gestures (Krauss et al., 1996). Johansson (1973) found that our visual perception of biological motion is keen enough to identify gestures that are made up of only 10 to 12 bright spots representing different human body joints. Prior literature has suggested that technologies designed with movable arms, hands, and/or bodies that imply intentions, motives, mental states, and social rituals can evoke our social perception (Salem et al., 2013). For instance, letting users shake hands with the robot NAO before negotiating with it led to more cooperation between users and robots (Bevan \& Fraser, 2015).

The other two primary cues, human-sounding voice and human-or animal-like shape, have already received much attention in the field of HMC. Many studies have demonstrated that human-sounding voice, whether actual or synthetic, has greater effects than machine-sounding speech in evoking social responses (Chérif \& Lemoine, 2019; Chiou et al., 2020; Xu, 2019). The human voice has especially been perceived as a natural and powerful cue and can more easily encourage individuals' learning performances compared to a machine voice (Mayer, 2014; Nass \& Brave, 2005). Interacting with Siri is an example of how a technology paired with mere vocal cues can raise individuals' involuntary awareness of a social entity. Fortunati (2003) used the phrase "the body reaches where the voice does" to highlight the idea that voice serves as an extension of the body in our daily interaction (p. 62).

Designing humanlike or animal-like shape into technologies has been effective in forging an attractive appearance. For instance, Pixar's mascot Luxo is designed to have a head and a body to increase its popularity. Martini et al. (2016) found that the degree of robots' humanlike appearance has a positive relationship with individuals' attribution of intentionality to the robots. Hinds et al. (2004) found that participants felt less responsible for a 
task when collaborating with a humanlike robot than with a machinelike robot partner, meaning that participants attributed more trust to the humanlike one than the machinelike one. Using fMRI, research has further suggested that even observing animal-like shadows created by finger movements on a screen activates the same brain activity as verbal communication, implying that perceiving animal shadows involves the same physiological reaction as decoding human speech (Fadiga et al., 2006).

One might argue that humans shifting from acceptance to revulsion when a technology appears highly humanlike but falls short of a perfect human replica (Mori et al., 2012) counters the classification of humanlike shape as a primary cue. While it is true that users' affinity for the machine may plunge at the occurrence of this "uncanny valley," experiencing eeriness itself is a manifestation of strong medium-as-social-actor presence, as it shows that humans develop strong emotional responses to a machine that approximates human appearances. Mori et al. (2012) postulated that the eerie sensation could be due to the human instinct that protects us from potential dangers, including members of different species appearing to be humanlike, which supports the idea that our responses to humanlike technologies are sensitive and intuitive.

\section{Secondary Cues}

Compared to primary cues that evoke evolutionary-based responses, secondary cues have less power in activating users' social perception and responses. Below we identify a few representative examples of secondary cues.

Whereas human or animal size could be a contributing factor to users' social responses to technologies (Duffy, 2003; Takayama \& Pantofaru, 2009), it is not always evocative. Walters et al. (2009) found that while in single trials a short robot led participants to allow for a closer approach distance than a taller robot, the effect faded away in repeated trials over three weeks. Additionally, when explaining the uncanny valley effects, Mori et al. (2012) used the Bunraku puppet to indicate that audiences tend to ignore the size of the puppet but concentrate on its appearances.

As a basic human means of exchanging social information (Dunbar, 2004), language use has been found to trigger social perception and responses (Sah \& Peng, 2015; Xu, 2020). However, it is classified as a secondary cue here because it does not always lead to strong social responses. For instance, a water bottle with the label "drink me" may induce greater levels of social reactions than one with the label "drink it." Thus, the effects depend on the variations in language. Informal language, warm (i.e., friendly and conversational) language, and language with vocal fillers and self-referential statements have been found to be more evocative than formal language, cold language, and language without paralinguistic cues (Goble \& Edwards, 2018; Hoffmann et al., 2020; Sah \& Peng, 2015).

Motion attracts our attention and may influence our social responses (Reeves \& Nass, 2000). The Heider-Simmel (1944) experiment suggests that even simple dots that randomly move on a computer screen can be interpreted to have intentions. So can different forms of automatic doors that move with different speeds and trajectories (Ju \& Takayama, 2009). However, movements have strong effects on social perception only when they become symbolic or conversational (Krauss et al., 1996). That is, movements that reveal communicators' 
purposes, mental states, and adherence to social rituals are more likely to evoke social reactions than random movements (Hoffman \& Ju, 2012; Xu, 2019). For instance, Fiore et al. (2013) found that an iRobot Ava that gave way to participants as it traveled across a hallway evoked stronger social presence than one that did not yield to participants. Thus, depending on their speed, trajectory, frequency, and social meanings, a technology's motion cues may trigger different levels of social perception (Mori et al., 2012).

Compared to a human-sounding voice, a machine-sounding voice is generally perceived as unnatural and unpleasant (Gong \& Lai, 2003). A machine-sounding voice has generally been found to be inferior to a human voice in evoking medium-as-social-actor presence experience (Chérif \& Lemoine, 2019). Moreover, the level of social responses evoked by a machine's voice, or other symbolic sounds, depends on the relationship between the technology and its users. In our daily interaction with technologies, alarm system voices; smartphone ringtones; SMS, calendar, and other alerts and reminders; the myriad sounds built into Windows, iOS, and other computer operating systems; and even the ticking and chiming of mechanical clocks all suggest the potential interpretation of a social actor for users who have come to understand, be comfortable with, and rely on them.

\section{Social Signals}

Beyond the primary and secondary cues, technologies can present social cues that can be interpreted as social signals in HMC (Fiore et al., 2013; Streater et al., 2012). That is, single or combinations of primary and/or secondary social cues can constitute abstract human characteristics including personalities, identities, and so on. Perceiving these social signals may require prolonged exposure to or interaction with the technologies. Contextual factors may also help individuals sense these abstract human characteristics. Below we provide some examples of social signals that are composed of social cues.

The ability of a media technology to be responsive or interactive should be sufficient on its own to trigger users' social perception and responses (Kim \& Sundar, 2012). But presenting interactivity requires technologies to demonstrate a constellation of cues, which may include a physically embodied robot's eye contact, nodding, and smiling, or a chatbot's timing of response, message contingency, and so on (Jung et al., 2014; Lew et al., 2018).

Perceived personality and social identity of a media technology also requires a constellation of cues. A systematic review (Mou et al., 2020) suggests that personalities of machines can be operationalized via cues such as speech styles, vocal features, movements, and proximity. In addition, social identity can be easily perceived based on minimal, but meaningful, cues. For instance, Eyssel and Kuchenbrandt (2012) assigned different names to two robots, one German name and one Turkish name. Participants in the group with the samenationality robot were more likely to evaluate the robot's performances as positive (Hogg \& Abrams, 1988; Kuchenbrandt et al., 2013).

Some people (and animals) in our lives become our companions, and some media technologies (e.g., Tamagotchi, books, smartphones) can provide the same sense of social connection. Early uses and gratifications research suggested that people's ritualized use of television is related to higher affinity with the television itself rather than the content it presents (Rubin, 1983). The physical presence of a technology itself is an essential cue and 
the additions of several others (e.g., sound, touch) can enhance the perceived social companionship.

All human beings are unique; aside from genetic and physical differences we all have different life experiences (Orwig, 2014; Turkle, 2012). We associate uniqueness with other people and value it in our interpersonal relationships (Eastwick \& Hunt, 2014). Many technologies can also be perceived as being unique, either because they come to us with these features or are designed or adapted for personalization. For example, many personal and mobile computers, virtual assistants, and other technologies ask users to provide a name for them; some people install custom ringtones and "skins" for electronic devices, and location-based mobile systems can provide customized context-aware information to assist users with decision-making (Choi et al., 2017). Even iRobot's Roomba vacuum cleaners can be personalized with a "skin toolkit" to increase users' acceptance of the device and their commitment to use it more (Sung et al., 2009).

A combination of social cues may cause individuals to infer the health status or the life span of technologies (Lechelt et al., 2020). When an old technology (e.g., mechanical clock, television, computer, smartphone, robot, etc.) begins to wear out or falter or is damaged in an accident, the owner may take it to a "doctor" (repair person) and wait with worry and impatience for a report on whether and how it will "recover." Even when technologies lose their functional value, or the developers stop supporting their use (e.g., the shutdown of servers that supported the Jibo Robot), the owners of the technologies may still find social value in keeping them as companions or for reimagined uses (see Lechelt et al., 2020).

Other cues and signals that could evoke medium-as-social-actor presence and social responses include olfactory cues (Chen, 2006), haptic cues (Blakemore, 2016; Li et al., 2017), interpersonal distance (Syrdal et al., 2006; Takayama \& Pantofaru, 2009; Walters et al., 2009), and degree of flexibility (Duffy \& Zawieska, 2012). It should be noted that even within a single cue, there exists significant components that evoke different effects. For example, vocal cues contain volume, pitch, tone, vocal outbursts, and so forth (Vinciarelli et al., 2009).

\section{Individual Differences and Contextual Factors}

We have suggested that social cues differ in their power over users' social responses. These social cues can further be converted into social signals that act as perceived human characteristics such as personalities, identities, and so on. As part of the MASA paradigm, here we further suggest the importance of individual differences and contextual factors that likely play a role in users' medium-as-social-actor presence and social responses.

Anthropocentrism is "the tendency of individuals to perceive the world from a human-centered perspective, in which humankind is the most significant of all entities" (Nass et al., 1995, p. 229). A person high in anthropocentrism is less likely to believe that technologies can, and should, take on physical and psychological attributes of people and occupy human social roles, which would likely suppress their medium-as-social-actor presence.

The CASA research has suggested that individuals' own personalities play a role when interacting with technologies; for example, a robot that demonstrated a complementary personality (Lee et al., 2006). Related to users' personalities, individuals with greater desire 
for social interaction are more likely to modulate their presence experiences by looking for social cues (Gardner et al., 2005) and perceiving social actors when they use technologies (Epley et al., 2007).

Epley et al. (2007) argued that children are more likely than adults to ascribe human emotions to external entities (e.g., angry clouds, happy sun). Flavell et al. (1990) found that children are more likely to perceive mediated actors or objects as "real." The same logic applies to medium-as-social-actor presence. In her book Alone Together, Turkle (2012) observed children's interaction with the toy Furby and noticed that children believe that Furby can burp, understand language, and may one day "die." All these observations suggest that age is an influential factor in our responses to technologies (Edwards et al., 2019).

Beyond these factors, individuals differ in their knowledge about, experience with, and ability to think critically about technologies, which should lead to different levels of medium-as-social-actor presence. For example, Nass and Moon (2000) suggested there was no evidence that computer experts are immune to social responses to computers. Johnson and colleagues (2004) found that those with more computer use experiences reported more positive affect in response to a computer's flattery and more favorable judgments of the computer. In addition, Lee (2010) found that people who were more analytical and rational were less likely to respond to flattery effects of computers compared to those who were intuitive and experiential.

Prior literature has suggested the potential of many other individual differences in leveraging our medium-as-social-actor presence experiences. For example, Salem et al. (2013) found that participants preferred robots that occasionally performed incorrect gestures over those that always performed perfect ones, implying that our tolerance of imperfection may help determine our social attitudes toward technologies. For people who have unrealistically high expectations for the performance of technologies, every disappointment highlights the true nature of the technologies, so these people will be less likely to have strong social responses to them (Paepcke \& Takayama, 2010; Waddell, 2018). Likewise, individuals' willingness to suspend disbelief may determine whether we treat a machine more as a tool or as a social entity (Duffy \& Zawieska, 2012). Moreover, individuals with different attachment styles diverge in their intention to seek social cues and form social relationships with other social actors (Cole \& Leets, 1999; Epley et al., 2007). Other relevant individual differences include gender, self-esteem, tolerance of uncertainty, tendency to make attribution errors, and so on (Epley et al., 2007; Nass et al., 1995; Rosen \& Knäuper, 2009).

As with other types of presence (Slater \& Wilbur, 1997; Won et al., 2015), a wide variety of factors related to the context of our exposure to and interactions with technologies may impact our experiences of medium-as-social-actor presence. These include, but are by no means limited to, the nature of the activity or task involved, the setting (e.g., public or private), the number of people present and our relationships with them, the amount and accuracy of information available to us regarding the nature of the technology, and even the time of day. More broadly, aspects of our culture are important factors (Bartneck et al., 2007). These include the prevailing attitudes toward technologies such as robots assuming social roles (Nass et al., 1995), the degree of industrialization that provides exposure to various technologies (Epley et al., 2007), the culture's tolerance of privacy invasion and data sharing (Nitto et al., 2017), and the subtle ways languages not only reflect but potentially guide our perceptions. To illustrate the power of language norms, note that in English at 
least, clocks have "faces" and "hands," chairs have "arms," needles have "eyes," streets have "shoulders," and computers and many other technologies can "die" (if we don't "kill" them when they don't "cooperate").

\section{MASA Mechanisms: Unifying Two Explanations}

So far we have presented the roles of primary cues, secondary cues, and social signals in the MASA paradigm. And we've discussed how, along with these factors, individual differences and contextual elements may further moderate the breadth and depth of users' social responses. Below we discuss two major explanatory mechanisms that have been supported in prior literature on CASA: mindlessness and anthropomorphism. Instead of viewing the two mechanisms as competing explanations for users' social perceptions and responses, we suggest in the MASA paradigm that these two mechanisms can be unified and account for different social response scenarios.

Mindlessness refers to the explanation that people are naturally oriented to the social rather than the asocial cues of technologies (Langer, 2000; Nass \& Moon, 2000). This may be based on evolutionarily based cognitive traits or learned from cues having been demonstrated repetitively in interpersonal communication, so that individuals "mindlessly (and) prematurely commit to overly simplistic scripts drawn in the past" (Nass \& Moon, 2000, p. 83). In contrast, anthropomorphism refers to a more active phenomenon, "the tendency to imbue the real or imagined behavior of nonhuman agents (e.g., animals, nature, gods, and mechanical or electronic devices) with humanlike characteristics, motivations, intentions, or emotions" (Epley et al., 2007, p. 864).

While both mindlessness and anthropomorphism seem to be logical explanations for people's social perception and responses, both of them have been found to have limitations. Regarding mindlessness for instance, Fischer and colleagues (2011) noticed that some participants laughed when receiving a robot's greetings, indicating that they found something odd and amusing about the interaction with the robot. Mou and $\mathrm{Xu}$ (2017) found that participants demonstrated two different personalities in interactions with chatbots versus humans. These studies suggest that users' responses to machines are not as mindless and spontaneous as to humans. Anthropomorphism cannot account for all CASA findings either. For example, Lee (2010) found that compared to text-only conditions, computer interfaces with anthropomorphic cartoon characters enhanced the social attractiveness and the trustworthiness of the computer, but they did not amplify flattery effects, which challenges the prediction that more anthropomorphic characters would facilitate stronger social responses (Lee, 2010).

The two explanatory mechanisms are seemingly antagonistic, as mindlessness occurs "without extensive thought or deliberation" (Moon, 2000, p. 325), and anthropomorphism "involves the thoughtful, sincere belief that the object has human characteristics" (Nass \& Moon, 2000, p. 93). However, a closer investigation into the role of technology cues may parse out the interrelationship between them. Specifically, Nass and Moon (2000) argued that to elicit mindlessness, an object must exhibit "enough cues" to bring forth social responses (p. 83), which implies that mindlessness may not occur when the cues are not evident or sufficient. On the other hand, anthropomorphism emphasizes the ascription of human mental or emotional states to nonhuman agents ranging from imagined ghosts to 
computer-generated dots (Duffy, 2003; Morewedge et al., 2007), which implies that even when objects are not designed with social cues (e.g., dots, clouds), anthropomorphism may occur as humans can mindfully assign human attributes to these nonhuman agents. Considering that Kim and Sundar (2012) noted that anthropomorphism can occur mindlessly or mindfully, we use mindless anthropomorphism and mindful anthropomorphism to unify the explanations for medium-as-social-actor presence and social responses. Specifically, mindless anthropomorphism should have more explanatory power when media technologies display cues that are of high quantity and high quality. In other words, if technologies demonstrate a group of primary social cues at the same time (e.g., Ishiguro's Gemonoid [Nishio et al., 2007] or Samsung's virtual human Neon [Vincent, 2020)]), users may not help having mindless, intuitive, and spontaneous responses to the technologies as these cues are natural, nuanced, and powerful. By contrast, when people experience strong medium-as-social-actor presence with technologies that are sufficient in neither the quality nor the quantity of cues, mindful anthropomorphism should be better at explaining the experience, as such scenarios indicate that individuals deliberately and thoughtfully attribute human characteristics to these technologies with limited cues (e.g., children imagining that their toys can speak to each other, drivers assigning names to their cherished cars) (see Figure 1).

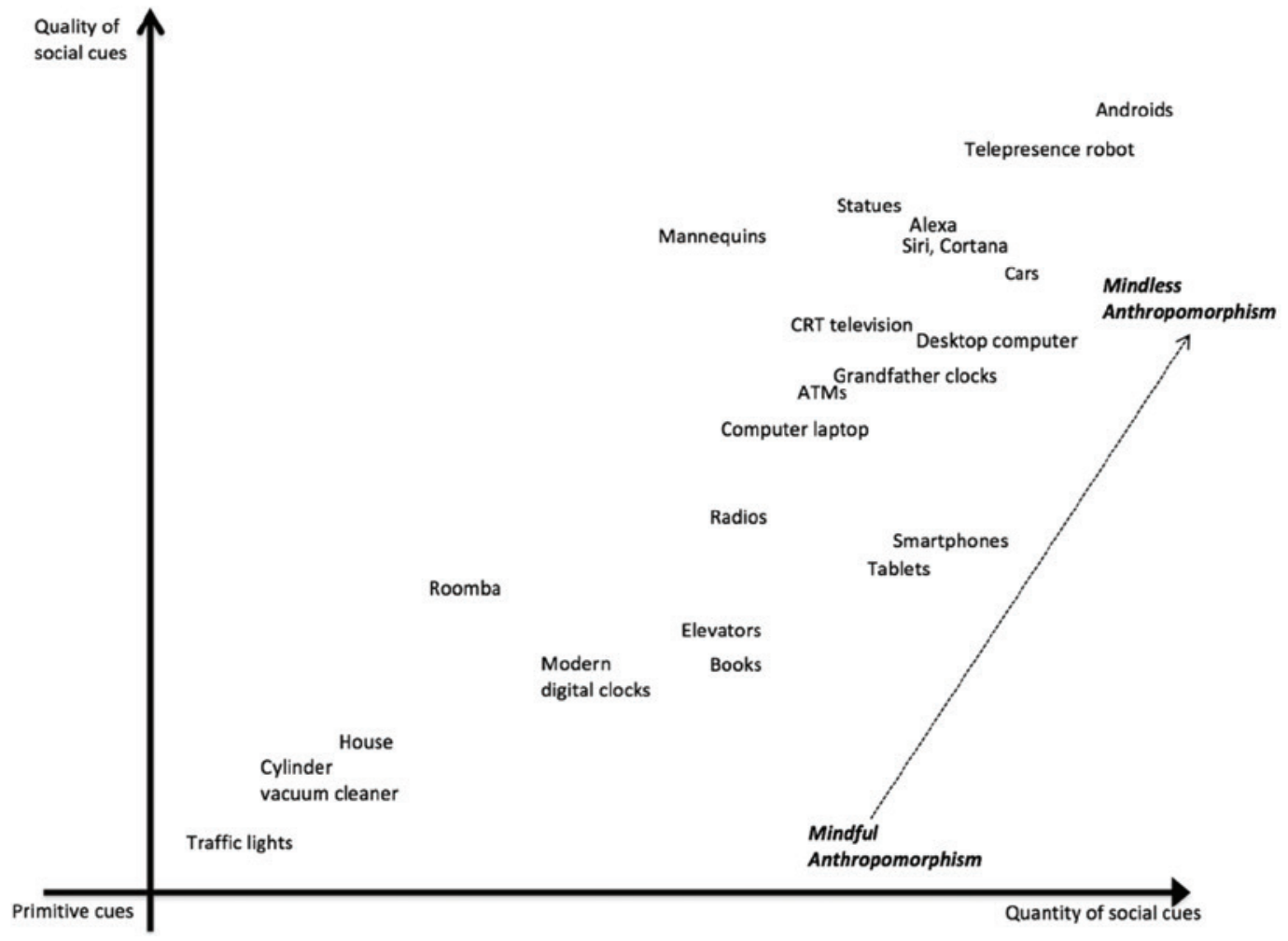

FIGURE 1 The Relationships Among Social Cues, Technologies, and Explanatory Mechanisms

Note: The placement of technologies in this figure represents only an approximation of the characteristics of prototypical examples of each technology. 
The distinction between mindless and mindful anthropomorphism may be useful in understanding two related questions raised in CASA. The first question stems from the fact that although Nass and Moon (2000) endorsed the mindlessness explanation, CASA "fails to pinpoint precisely when and why mindless behavior will occur" (Nass \& Moon, 2000, p. 96). Our mapping of mindless anthropomorphism and mindful anthropomorphism provides a reasonable, literature-based, and testable contention about which mechanism may be more explanatory depending on the nature and number of technology cues available to users.

The second question is related to the CASA assumption that technologies must demonstrate enough cues to elicit mindless social responses (Nass \& Moon, 2000). What qualifies as enough, or in other words, how social the cues need to be to induce mindless responses, is unclear. Our categorization of cues as primary and secondary, along with the scope of application of mindless versus mindful anthropomorphism, provides a testable suggestion that in order to evoke mindless responses, the cues should ideally be sufficient in both quality and quantity. While research will need to establish the relative impacts of different combinations of high quality (primary) cues and secondary cues, the definition of primary cues suggests that quality is of paramount importance.

As with the effects of cues on medium-as-social-actor presence and social responses, the activation of mindless versus mindful processing is contingent upon a series of factors including the specific combination of cues, individual differences, and contextual factors. Thus, it is possible that some individuals may mindlessly respond to media with fewer social cues or mindfully respond to media that demonstrate more social cues. This is consistent with Fischer's (2011) speculation that individuals differ in their inclination to be mindless and Fussel and colleagues' (2008) finding that users demonstrate different degrees of anthropomorphism in their spontaneous responses to actual robots versus their carefully considered conceptions of robots.

We also readily acknowledge that other possible explanations for social responses that have received less attention in the CASA literature may become more useful in explaining our increasingly complicated and diverse media use practices. These include the source orientation explanation (Solomon \& Wash, 2014), cognitive load explanation (Lee, 2008), and folk explanations of behavior (Malle, 1999).

\section{Evolutionary Foundation and Propositions of the MASA Paradigm}

Above we have differentiated primary cues from secondary cues and parsed out the relationship between mindless and mindful anthropomorphism. Below we first suggest how the MASA paradigm and the social perceptions and responses it seeks to predict and explain are established upon three inter-related interpretations of socialness. Then we formally list the propositions of the MASA paradigm based on the arguments presented here and above.

In the CASA literature and so far in this paper the term social is treated as a "primitive" term (Chaffee, 2009) with a meaning that is widely understood; essentially something is social if it involves human perceptions and behaviors in response to other humans. But given its role in CASA and MASA, it is important to be clear about what is meant by this term. Here we provide three related conceptualizations of socialness. All three are consistent 
with the precepts of evolutionary psychology that suggest we have developed our cognitive, affective, and behavioral abilities via natural selection (Brewer, 2004; Sokol \& Strout, 2007).

First, our brain is social. Theory of mind suggests that we can infer others' intentions, desires, and motivations based on their social cues (Adolphs, 2009; Banks, 2020; Frith \& Frith, 2005). By predicting others' intention and actions, humans can adjust their own actions accordingly, and more effectively communicate and collaborate with others (Hare, 2007). Research has shown that in this inference process, the amygdala, an important structure in the medial temporal lobe of our brain, is activated to regulate our social perception and behavior (Adolphs, 2009). The tenet of theory of mind is consistent with the idea of egocentric modeling (Epley et al., 2007), which suggests that humans can only experience the world from their own perspective. Although we can speculate about others' intentions, we may make false predictions and cannot know exactly what it is like to be another animate or inanimate entity. Given the importance of interpreting social cues and the inherent challenges we face in doing so, the notion of the social brain means that no matter with what object we interact, we are at least somewhat oriented to perceive the object as social.

The second interpretation of being social indicates that our way of living is social. Here, social means being interdependent and cooperative (Brewer, 2004; Tomasello, 2014). Based on the perspective of adaptation, to resist environmental risks and avoid potential threats, primitives select to live in groups, make tools, and collaborate with each other. These means of coordination and collaboration have acted as a buffer between the individuals and actual and potential ecological changes (Caporael \& Brewer, 1995). The instinct to avoid dangers is so imprinted in our adaptation that today we are still "hardwired" to monitor the world around us for threats (Shoemaker, 1996). It is consistent with Reeves and Nass's (1996) explanation that our brain has not evolved to distinguish mediated objects and nonmediated objects. It is also aligned with the findings regarding arousal and attention patterns in television viewers (Reeves \& Thorson, 1986) - at some level viewers respond to movement and change even if the potential threat is only shown on an electronic display screen.

These two interpretations of humans' social orientation can help us understand how users can perceive technologies with even limited cues, including technologies not created or used for communication (i.e., not media technologies), as social. For example, imagine a person who sees a hammer for the first time and needs to figure out how to use it. Although a hammer serves primarily a simple, functional purpose, it can be perceived as social in that first, observing the shape of the hammer, feeling the weight of the head, and finding out that the handle is designed to be held by one hand, is a process of using minimal visual and haptic cues to predict the potential use of the technology. And second, using the hammer to strike other objects or pull out nails can be viewed as an interdependence between the user and the tool to accomplish a goal. Using technology is a form of collaboration that in our long history humans have enacted to attempt to improve our living conditions. So even though a technology may present limited cues and be designed for a simple purpose, it retains a subtle social element that we are hardwired to perceive.

The third interpretation of being social refers to our being adaptive and flexible. Research has found that although humans are not the only animals that infer each other's mental states, humans have evolved to develop the propensity for flexibility in using social cues during communication and cooperation with others, which is less evident for animals 
like chimpanzees (Hare, 2007). Demonstrating flexibility across different scenarios is likely to precipitate humans' cooperation process. This evolutionary characteristic is also inherent in our interaction with technologies. Some may argue if a study discovers that humans do not treat machines as humans, the study refutes the CASA proposition. However, the MASA paradigm holds that our social responses to technologies vary just as our social responses vary when we encounter and interact with children, foreigners, strangers, and friends (and among individuals within these categories) (Katagiri et al., 2001). As technologies transform to become multi-modal, ubiquitous, context-aware, and even invisible (Campbell, 2020; Fortunati, 1995; Ling, 2012), humans apply our ability to develop flexible and even unique social rules for interactions in all kinds of HMC scenarios. As an example, typing letters using a keyboard is a skill we learn so that we can interact with computers, but the behavior itself is ultimately a form of interdependence, which is considered the essence of being a social species (Caporael \& Brewer, 1995; Tomasello, 2014).

We have explicated what social means in the MASA paradigm. We have also advocated for distinguishing the quantity of social cues and the quality of social cues presented by technologies. In addition, we explained how mindless and mindful responses can be dialectically applied to different technology use scenarios. Below we present a set of propositions based on the theoretical and empirical evidence discussed above. Specifically, Proposition 1 is related to the evolutionary foundation of the MASA paradigm. Propositions 2 to 6 are related to the different effects of social cues. Propositions 7 to 9 are based on the relationship between mindless and mindful anthropomorphism.

P1: Every media technology has at least some potential to evoke medium-associal-actor presence and corresponding social responses.

P2: It is not only the social cues but also the combination of social cues, social signals, individual factors, and contextual factors that lead to medium-associal-actor presence and corresponding social responses.

P3: Some social cues are primary: Each is sufficient but not necessary to evoke medium-as-social-actor presence.

P4: Some social cues are secondary: Each is neither sufficient nor necessary to evoke medium-as-social-actor presence.

P5a: All other conditions being equal, individuals are more likely to experience medium-as-social-actor presence and socially respond to media technologies that display cues with more human characteristics (quality of cues).

P5b: All other conditions being equal, individuals are more likely to experience medium-as-social-actor presence and socially respond to media technologies that display more social cues (quantity of cues). 
P6: All other conditions being equal, the quality of cues (primary vs. secondary) has a greater role in evoking medium-as-social-actor presence and corresponding social responses than the quantity (number) of cues.

P7: Individuals vary in their tendency to perceive and respond to media technologies as social actors.

P8: Individuals' social responses to media technologies can occur with either mindless or mindful processing.

P9a: All other conditions being equal, media technologies that display more cues (quantity of cues) are more likely to lead individuals to mindlessly perceive them as social actors. Conversely, all other conditions being equal and given the same level of social responses, media technologies that display fewer cues (quantity of cues) are more likely to lead individuals to mindfully perceive them as social actors.

P9b: All other conditions being equal, media technologies that display cues with more human characteristics (quality of cues) are more likely to lead individuals to mindlessly perceive them as social actors. Conversely, all other conditions being equal and given the same level of social responses, media technologies that display cues with fewer human characteristics (quality of cues) are more likely to lead individuals to mindfully perceive them as social actors.

\section{Contributions, Scope, and Future Research}

The MASA paradigm follows the major tenet of the CASA paradigm and expands it to more media technologies, including emerging and future technologies. The MASA paradigm can make the following contributions. First, the original CASA paradigm and the Media Equation described a series of experiments on users' social responses to computers and televisions. They did not explicitly list what propositions scholars could rely on to derive additional research questions. The MASA paradigm lists nine propositions here so that future research can test, refine, and enhance the theoretical framework in its predictive power, explanatory power, falsifiability, heuristic value, and so on (Chaffee \& Berger, 1987).

Second, most prior research has primarily focused on the effects of single social cues or the cumulative effects of social cues, while the quality of social cues-whether they are central to our perception of socialness-has not been systematically examined. The MASA paradigm suggests that there exists a hierarchy of social cues that represents their potential to evoke social perceptions and responses. With knowledge about the quality of social cues, designers and developers may scrutinize different effects of social cues and find the optimal combination of cues to create positive use experiences.

Third, the paradigm identifies the roles of individual differences and contextual factors in generating users' medium-as-social-actor presence and social responses. It provides an analysis of the whole communication scenario in which individuals are perceivers, technologies are manifestations of social cues, and contexts guide perceptions and responses, with 
all of these elements influential in evoking medium-as-social-actor presence and social responses (see Figure 2). Future research can test the moderating roles of particular individual differences and contextual factors while investigating users' social reaction to different sets of technology cues.

Fourth, the paradigm has articulated an evolutionary base and explained what it means to have "social" perceptions and responses. Social responses do not necessarily equate to human responses. Social perceptions and social responses occur because we have a social brain, live a social life, and are used to making adaptations to be cooperative and interdependent. Based on this theoretical foundation, we have attempted to provide answers to Nass and Moon's (2000) questions about (1) what dimensions of technologies are more powerful in evoking social responses, (2) when mindless processing is more likely to occur, and (3) how "social" a technology should be to bring forth mindless social responses. We believe that the current MASA paradigm can be useful in future research on social responses to technologies and we encourage theoretical refinements in the paradigm that can bring greater understanding of human behaviors with respect to technology and make possible the practical benefits of improved product design and user experience.

Despite these contributions, some may wonder about the technologies to which the MASA paradigm applies and whether the term MASA could be replaced by TASA (i.e., Technologies are Social Actors). Here we refer to a technology as a "machine, device, or other application of human industrial arts" (ISPR, 2000, presence defined). We refer to media technologies as "artifacts or devices used to communicate or convey information" (Lievrouw \& Livingstone, 2006, p. 23). In explaining that the MASA paradigm is rooted in evolutionary psychology we noted that humans are at least somewhat oriented to perceive technologies as social. Therefore, as part of the MASA paradigm, we acknowledge that people's social responses may occur to all technologies (sometimes even beyond technologies), especially when people mindfully and consciously anthropomorphize those technologies that are not designed to be used for social purposes. As media and communication scholars, however, we concentrate more on media technologies, including both traditional and emerging ones such as books, televisions, computers, smartphones, tablets, and so on. More importantly, we call it the MASA paradigm because this term reflects a shift of focus

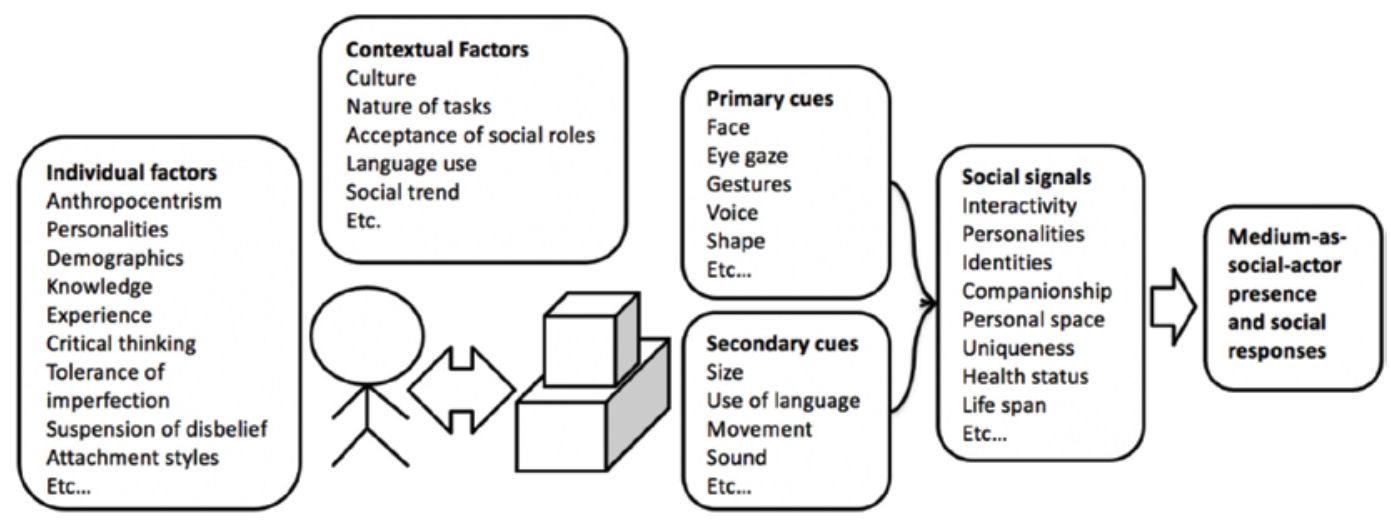

The Media are Social Actors Paradigm

FIGURE 2 The Media Are Social Actors paradigm 
from media as channels for message transmission to media as interlocutors or communicators (Gunkel, 2012). Although the role of media technologies may neither be entirely of a communication channel nor of a communication terminal in the current media landscape (Guzman, 2018), the MASA paradigm is targeted at the phenomena in which users perceive media technologies to be independent social entities, and partially or sometimes fully overlook their nature as mere machines or communication channels.

Several paths are available for researchers to investigate the validity of the MASA paradigm and refine and expand it. First, researchers should conduct empirical studies to verify the nine preliminary propositions. Studies can explore whether there exists a hierarchy of social cues that reliably elicit different degrees and/or particular types of social responses. Although few methods can provide direct evidence to investigate propositions that require differentiating users' mindless and mindful processing, researchers can use a combination of methods and measures, including both objective and subjective ones (e.g., fMRI and EEG versus interviews) and real-time and retrospective ones (e.g., secondary task reaction time and think aloud protocols versus post-exposure memory tests and questionnaires), to look for the convergence of results.

Second, researchers should look beyond CASA studies to test whether and how findings and predictions from other interpersonal communication theories and computermediated communication (CMC) theories can be adapted to the MASA paradigm. Some interpersonal communication theories researchers might examine include Communication Accommodation Theory (Giles, 2008), Communication Privacy Management Theory (Petronio \& Durham, 2008), Expectancy Violation Theory (Burgoon \& Hale, 1988), Uncertainty Management Theory (Bradac, 2001), Interpersonal Deception Theory (Burgoon et al., 2008), and the Theory of Imagined Interaction (Honeycutt, 2002). Some CMC theories or models that have been applied but could be explored further include the Social Identity model of De-individuation Effects (SIDE) (Reicher et al., 1995) and Social Information Processing Theory (Walther, 1996).

Third, researchers can further examine and add details to the psychological processes described in the MASA paradigm. For example, how do attention and memory influence medium-as-social-actor presence? What determines whether presence will lead to behavioral social responses? As researchers have not achieved consensus on the explanations for the phenomena captured in the CASA paradigm, more research should be conducted to account for the mental processes that underlie people's social perceptions of and responses to today's and tomorrow's diverse and evolving technologies.

Finally, researchers should explore and discuss with designers how they can apply the MASA paradigm and findings. Though most of the time richer media presentations can bring about better experiences, higher medium-as-social-actor presence (as with any type of presence) does not necessarily produce higher efficacy or popularity. Thus, designers should be fully aware of the advantages and disadvantages of their choices in applying the paradigm. They should also consider the larger ethical issues involved in creating technologies that lead users to perceive and treat media as social actors. As it is possible to view users of these technologies as victims of deception, unconscious responses, and the manipulation of presence (Biocca et al., 2003; Lombard, 2009), designing and marketing technologies that evoke medium-as-social-actor-presence and social responses should be based on thoughtful, informed, and ethical analyses. 


\section{Author Biographies}

Matthew Lombard ( $\mathrm{PhD}$, Stanford University) is co-founder and president of the International Society for Presence Research (ISPR, http://ispr.info) and an associate professor in the Department of Media Studies and Production at Temple University in Philadelphia, Pennsylvania, USA. His research centers on individuals' psychological and physiological processing of media presentations and experiences, with particular focus on the concept of (tele)presence. His work has appeared in academic journals including Behaviour \& Information Technology, CyberPsychology and Behavior, Human Communication Research, Human Technology, Journal of Computer-Mediated Communication, Journal of Communication, and Presence: Teleoperators and Virtual Environments. For more detailed information please visit http://matthewlombard.com.

Kun $\mathrm{Xu}$ (PhD, Temple University) is an assistant professor of emerging media at University of Florida. His work focuses on the concepts of social cues and social presence in human-computer interaction, human-robot interaction, and computer-mediated communication.

(D) https://orcid.org/0000-0001-9044-821X

\section{References}

Abubshait, A., \& Wiese, E. (2017). You look human, but act like a machine: Agent appearance and behavior modulate different aspects of human-robot interaction. Frontiers in Psychology, 8, 1393. https://doi.org/10.3389/fpsyg.2017.01393

Adolphs, R. (2009). The social brain: Neural basis of social knowledge. Annual Review of Psychology, 60, 693-716.

Andrist, S., Mutlu, B., \& Tapus, A. (2015). Look like me: Matching robot personality via gaze to increase motivation. In Proceedings of the 33rd Annual ACM Conference on Human Factors in Computing Systems (pp. 3603-3612). https://doi.org/10.1145/2702123.2702592

Antheunis, M. L., \& Schouten, A. P. (2011). The effects of other-generated and systemgenerated cues on adolescents' perceived attractiveness on social network sites. Journal of Computer-Mediated Communication, 16(3), 391-406. https://doi.org/10.1111/j.10836101.2011.01545.x

Araujo, T. (2018). Living up to the chatbot hype: The influence of anthropomorphic design cues and communicative agency framing on conversational agent and company perceptions. Computers in Human Behavior, 85, 183-189. https://doi.org/10.1016/j. chb.2018.03.051

Banks, J. (2020). Theory of mind in social robots: Replication of five established human tests. International Journal of Social Robotics, 12(2), 403-414. https://doi.org/10.1007/ s12369-019-00588-x

Bartneck, C., Reichenbach, J., \& Carpenter, J. (2008). The carrot and the stick: The role of praise and punishment in human-robot interaction. Interaction Studies, 9(2), 179-203. https://doi.org/10.1075/is.9.2.03bar 
Bartneck, C., Suzuki, T., Kanda, T., \& Nomura, T. (2007). The influence of people's culture and prior experiences with Aibo on their attitude towards robots. AI and Society, 21(1), 217-230. https://doi.org/10.1007/s00146-006-0052-7

Bevan, C., \& Fraser, D. S. (2015). Shaking hands and cooperation in tele-present humanrobot negotiation. In Proceedings of the Tenth Annual ACM/IEEE International Conference on Human-Robot Interaction (pp. 247-254). IEEE.

Biocca, F., Harms, C., \& Burgoon, J. K. (2003). Toward a more robust theory and measure of social presence: Review and suggested criteria. Presence: Teleoperators \& virtual environments, 12(5), 456-480. https://doi.org/10.1162/105474603322761270

Blakemore, E. (2016). Touching robots can turn humans on, study finds. Washington Post. https://www.washingtonpost.com/news/speaking-of-science/wp/2016/04/05/touchingrobots-can-turn-humans-on-study-finds/

Bradac, J. J. (2001). Theory comparison: Uncertainty reduction, problematic integration, uncertainty management, and other curious constructs. Journal of Communication, 51(3), 456-476. https://doi.org/10.1111/j.1460-2466.2001.tb02891.x

Brewer, M. B. (2004). Taking the social origins of human nature seriously: Toward a more imperialist social psychology. Personality and Social Psychology Review, 8(2), 107-113. https://doi.org/10.1207/s15327957pspr0802_3

Burgoon, J. K., Blair, J. P., \& Strom, R. E. (2008). Cognitive biases and nonverbal cue availability in detecting deception. Human Communication Research, 34(4), 572-599. https://doi.org/10.1111/j.1468-2958.2008.00333.x

Burgoon, J. K., Bonito, J. A., Bengtsson, B., Cederberg, C., Lundeberg, M., \& Allspach, L. (2000). Interactivity in human-computer interaction: A study of credibility, understanding, and influence. Computers in Human Behavior, 16(6), 553-574.

Burgoon, J. K., \& Hale, J. L. (1988). Nonverbal expectancy violations: Model elaboration and application to immediacy behaviors. Communication Monographs, 55(1), 58-79. https://doi.org/10.1080/03637758809376158

Campbell, S. W. (2020). Cutting the cord: Social and scholarly revolutions as CMC goes mobile. Journal of Computer-Mediated Communication, 25(1), 101-110. https://doi. org/10.1093/jcmc/zmz021

Caporael, L. R., \& Brewer, M. B. (1995). Hierarchical evolutionary theory: There is an alternative, and it's not creationism. Psychological Inquiry, 6(1), 31-34. https://doi. org/10.1207/s15327965pli0601_2

Carolus, A., Muench, R., Schmidt, C., \& Schneider, F. (2019). Impertinent mobiles-Effects of politeness and impoliteness in human-smartphone interaction. Computers in Human Behavior, 93, 290-300. https://doi.org/10.1016/j.chb.2018.12.030

Chaffee, S. H. (2009). Thinking about theory. In D. W. Stacks \& M. B. Salwen (Eds.), An integrated approach to communication theory and research (pp. 27-43). Lawrence Erlbaum.

Chaffee, S. H., \& Berger, C. R. (1987). What communication scientists do. In C. R. Berger \& S. H. Chaffee (Eds.), Handbook of communication science (pp. 99-122). Sage.

Chérif, E., \& Lemoine, J. F. (2019). Anthropomorphic virtual assistants and the reactions of Internet users: An experiment on the assistant's voice. Recherche et Applications en Marketing, 34(1), 28-47. https://doi.org/10.1177/2051570719829432

Chen, Y. (2006). Olfactory display: Development and application in virtual reality therapy. In The 16th International Conference on Artificial Reality and Telexistence (pp. 580-584). 
Chiou, E. K., Schroeder, N. L., \& Craig, S. D. (2020). How we trust, perceive, and learn from virtual humans: The influence of voice quality. Computers \& Education, 146, 103756. https://doi.org/10.1016/j.compedu.2019.103756

Choi, B., Kwon, O., \& Shin, B. (2017). Location-based system: Comparative effects of personalization vs ease of use. Telematics and Informatics, 34(1), 91-102. https://doi. org/10.1016/j.tele.2016.04.011

Cole, T., \& Leets, L. (1999). Attachment styles and intimate television viewing: Insecurely forming relationships in a parasocial way. Journal of Social and Personal Relationships, 16(4), 495-511. https://doi.org/10.1177/0265407599164005

Duffy, B. R. (2003). Anthropomorphism and the social robot. Robotics and Autonomous Systems, 42, 177-190. https://doi.org/10.1016/S0921-8890(02)00374-3

Duffy, B. R., \& Zawieska, K. (2012). Suspension of disbelief in social robotics. In The 21st IEEE International Symposium on Robot and Human Interactive Communication (pp. 484-489). IEEE.

Dunbar, R. I. M. (2004). Gossip in evolutionary perspective. Review of General Psychology, 8, 100-110. https://doi.org/10.1037/1089-2680.8.2.100

Eastwick, P. W., \& Hunt, L. L. (2014). Relational mate value: Consensus and uniqueness in romantic evaluations. Journal of Personality and Social Psychology, 106(5), 728-751. https://doi.org/10.1037/a0035884

Edwards, C., Edwards, A., Stoll, B., Lin, X., \& Massey, N. (2019). Evaluations of an artificial intelligence instructor's voice: Social identity theory in human-robot interactions. Computers in Human Behavior, 90, 357-362. https://doi.org/10.1016/j.chb.2018.08.027

Epley, N., Waytz, A., \& Cacioppo, J. T. (2007). On seeing human: A three-factor theory of anthropomorphism. Psychological Review, 114(4), 864-886. https://doi. org/10.1037/0033-295X.114.4.864

Eyssel, F., \& Kuchenbrandt, D. (2012). Social categorization of social robots: Anthropomorphism as a function of robot group membership. British Journal of Social Psychology, 51(4), 724-731. https://doi.org/10.1111/j.2044-8309.2011.02082.x

Fadiga, L., Craighero, L., Destro, M. F., Finos, L., Cotillon-Williams, N., Smith, A. T., \& Castiello, U. (2006). Language in shadow. Social Neuroscience, 1(2), 77-89. https://doi. org/10.1080/17470910600976430

Fink, B., \& Penton-Voak, I. (2002). Evolutionary psychology of facial attractiveness. Current Directions in Psychological Science, 11(5), 154-158. https://doi.org/10.1111/14678721.00190

Fiore, S. M., Wiltshire, T. J., Lobato, E. J. C., Jentsch, F. G., Huang, W. H., \& Axelrod, B. (2013). Toward understanding social cues and signals in human-robot interaction: Effects of robot gaze and proxemic behavior. Frontiers in Psychology, 4, 1-15. https:// doi.org/10.3389/fpsyg.2013.00859

Fischer, K. (2011). Interpersonal variation in understanding robots as social actors. In The 2011 6th ACM/IEEE International Conference on Human-Robot Interaction (HRI) (pp. 53-60).

Fischer, K., Foth, K., Rohlfing, K., \& Wrede, B. (2011). Mindful tutors: Linguistic choice and action demonstration in speech to infants and a simulated robot. Interaction StudiesSocial Behaviour and Communication in Biological and Artificial Systems, 12(1), 134161. https://doi.org/10.1075/is.12.1.06fis 
Fiske, S. T., \& Taylor, S. E. (1991). Social cognition. McGraw-Hill.

Flavell, J. H., Flavell, E. R., Green, F. L., \& Korfmacher, J. E. (1990). Do young children think of television images as pictures or real objects? Journal of Broadcasting \& Electronic Media, 34(4), 399-419. https://doi.org/10.1080/08838159009386752

Fortunati, L. (1995). Gli italiani al telefono [Italians at the phone]. Angeli.

Fortunati, L. (2003). Real people, artificial bodies. In L. Fortunati, J. E. Katz, \& R. Riccini (Eds.), Mediating the human body: Technology, communication, and fashion (pp. 61-74). Lawrence Erlbaum Associates, Inc.

Frith, C., \& Frith, U. (2005). Theory of mind. Current Biology, 15, 644-645.

Fussell, S. R., Kiesler, S., Setlock, L. D., \& Yew, V. (2008). How people anthropomorphize robots. In 3rd ACM/IEEE International Conference on Human-Robot Interaction (pp. 145-152).

Gambino, A., Fox, J., \& Ratan, R. (2020). Building a stronger CASA: Extending the computers are social actors paradigm. Human-Machine Communication, 1, 71-86. https://doi. org/10.30658/hmc.1.5

Gardner, W. L., Pickett, C. L., Jefferis, V., \& Knowles, M. (2005). On the outside looking in: Loneliness and social monitoring. Personality and Social Psychology Bulletin, 31(11), 1549-1560. https://doi.org/10.1177/0146167205277208

Gauthier, I., \& Tarr, M. J. (1997). Becoming a "Greeble" expert: Exploring mechanisms for face recognition. Vision Research, 37(12), 1673-1682. https://doi.org/10.1016/S00426989(96)00286-6

Ghazali, A. S., Ham, J., Barakova, E., \& Markopoulos, P. (2018). The influence of social cues in persuasive social robots on psychological reactance and compliance. Computers in Human Behavior, 87, 58-65. https://doi.org/10.1016/j.chb.2018.05.016

Giles, H. (2008). Communication accommodation theory. Sage Publications.

Goble, H., \& Edwards, C. (2018). A robot that communicates with vocal fillers has... Uhhh... greater social presence. Communication Research Reports, 35(3), 256-260. https://doi.org/10.1080/08824096.2018.1447454

Gong, L. (2008). How social is social responses to computers? The function of the degree of anthropomorphism in computer representations. Computers in Human Behavior, 24(4), 1494-1509. https://doi.org/10.1016/j.chb.2007.05.007

Gong, L., \& Lai, J. (2003). To mix or not to mix synthetic speech and human speech? Contrasting impact on judge-rated task performance versus self-rated performance and attitudinal responses. International Journal of Speech Technology, 6(2), 123-131. https:// doi.org/10.1023/A:1022382413579

Gunkel, D. J. (2012). Communication and artificial intelligence: Opportunities and challenges for the 21st century. Communication+ 1, 1(1), 1-25. https://doi.org/10.7275/ R5QJ7F7R

Guzman, A. L. (Ed.). (2018). Human-machine communication: Rethinking communication, technology, and ourselves. Peter Lang Publishing, Incorporated.

Hare, B. (2007). From nonhuman to human mind: What changed and why? Current Directions in Psychological Science, 16(2), 60-64. https//doi.org/10.1111/j.1467-8721.2007. 00476.x

Heider, F., \& Simmel, M. (1944). An experimental study of apparent behavior. The American Journal of Psychology, 57(2), 243-259. https://doi.org/10.2307/1416950 
Hinds, P. J., Roberts, T. L., \& Jones, H. (2004). Whose job is it anyway? A study of humanrobot interaction in a collaborative task. Human-Computer Interaction, 19(1), 151-181. https://doi.org/10.1207/s15327051hci1901\&2_7

Hoffman, G., \& Ju, W. (2012). Designing robots with movement in mind. Journal of Human Robot Interaction, 1(1), 78-95. https://doi.org/10.5898/JHRI.3.1.Hoffman

Hoffmann, L., Derksen, M., \& Kopp, S. (2020, March). What a pity, Pepper! How warmth in robots' language impacts reactions to errors during a collaborative task. In 2020 ACM/ IEEE International Conference on Human-Robot Interaction (pp. 245-247). https://doi. org/10.1145/3371382.3378242

Hogg, M. A., \& Abrams, D. (1988). Social identifications: A social psychology of intergroup relations and group processes. Routledge.

Honeycutt, J. M. (2002). Imagined interactions: Daydreaming about communication. Hampton Pr.

Horton, D., \& Wohl, R. R. (1956). Mass communication and para-social interaction. Observations on intimacy at a distance. Psychiatry, 19, 215-229. https://doi.org/10.1080/0033 2747.1956.11023049

International Society for Presence Research (ISPR). (2000). The concept of presence: Explication statement. ISPR. https://ispr.info/about-presence-2/about-presence/

Johansson, G. (1973). Visual perception of biological motion and a model for its analysis. Perception \& Psychophysics, 14(2), 201-211. https://doi.org/10.3758/BF03212378

Johnson, D., Gardner, J., \& Wiles, J. (2004). Experience as a moderator of the media equation: The impact of flattery and praise. International Journal of Human Computer Studies, 61(3), 237-258. https://doi.org/10.1016/j.ijhcs.2003.12.008

Ju, W., \& Takayama, L. (2009). Approachability: How people interpret automatic door movement as gesture. International Journal of Design, 3(2), 1-10.

Jung, M. F., Martelaro, N., Hoster, H., \& Nass, C. (2014). Participatory materials: Having a reflective conversation with an artifact in the making. In Proceedings of the 2014 Conference on Designing Interactive Systems (pp. 25-34). https://doi.org/10.1145/ 2598510.2598591

Kanda, T., Shiomi, M., Miyashita, Z., Ishiguro, H., \& Hagita, N. (2009, 2008). An affective guide robot in a shopping mall. In Proceedings of the 4th ACM/IEEE International Conference on Human Robot Interaction (pp. 173-180). https://doi.org/10.1145/1514095. 1514127

Katagiri, Y., Nass, C., \& Takeuchi, Y. (2001). Cross-cultural studies of the computers are social actors paradigm: The case of reciprocity. In M. Smith, G. Salvendy, \& D. Harris (Eds.), Usability evaluation and interface design: Cognitive engineering, intelligent agents, and virtual reality (pp. 1558-1562). Lawrence Erlbaum.

Kim, Y., \& Sundar, S. S. (2012). Anthropomorphism of computers: Is it mindful or mindless? Computers in Human Behavior, 28(1), 241-250. https://doi.org/10.1016/j.chb.2011.09.006

Kobiella, A., Grossmann, T., Reid, V. M., \& Striano, T. (2008). The discrimination of angry and fearful facial expressions in 7-month-old infants: An event-related potential study. Cognition and Emotion, 22(1), 134-146. https://doi.org/10.1080/02699930701394256

Krauss, R. M., Chen, Y., \& Chawla, P. (1996). Nonverbal behavior and nonverbal communication: What do conversational hand gestures tell us? Advances in Experimental Social Psychology, 28(C), 389-450. https://doi.org/10.1016/S0065-2601(08)60241-5 
Kuchenbrandt, D., Eyssel, F., Bobinger, S., \& Neufeld, M. (2013). When a robot's group membership matters. International Journal of Social Robotics, 5(3), 409-417. https://doi. org/10.1007/s12369-013-0197-8

Langer, E. J. (2000). Mindful learning. Current Directions in Psychological Science, 9(6), 220-223. https://doi.org/10.1111/1467-8721.00099

Lechelt, S., Gorkovenko, K., Soares, L. L., Speed, C., Thorp, J. K., \& Stead, M. (2020). Designing for the end of life of IoT objects. In Companion Publication of the 2020 ACM Designing Interactive Systems (pp. 417-420).

Lee, E. J. (2008). Gender stereotyping of computers: Resource depletion or reduced attention? Journal of Communication, 58(2), 301-320. https://doi.org/10.1111/j.14602466.2008.00386.x

Lee, E. J. (2010). What triggers social responses to flattering computers? Experimental tests of anthropomorphism and mindlessness explanations. Communication Research, 37(2), 191-214. https://doi.org/10.1177/0093650209356389

Lee, K. M., Peng, W., Jin, S. A., \& Yan, C. (2006). Can robots manifest personality?: An empirical test of personality recognition, social responses, and social presence in human-robot interaction. Journal of Communication, 56(4), 754-772. https://doi. org/10.1111/j.1460-2466.2006.00318.x

Lew, Z., Walther, J. B., Pang, A., \& Shin, W. (2018). Interactivity in online chat: Conversational contingency and response latency in computer-mediated communication. Journal of Computer-Mediated Communication, 23(4), 201-221. https://doi.org/10.1093/ jcmc/zmy009

Li, J. J., Ju, W., \& Reeves, B. (2017). Touching a mechanical body: Tactile contact with body parts of a humanoid robot is physiologically arousing. Journal of Human-Robot Interaction, 6(3), 118-130. https://doi.org/10.5898/jhri.6.3.li

Lievrouw, L. A., \& Livingstone, S. (2006). Handbook of new media: Social shaping and consequences of ICTs. Sage.

Ling, R. (2012). Taken for grantedness: The embedding of mobile communication into society. MIT Press.

Lombard, M. (2009). The promise and peril of telepresence. In C. Bracken \& P. Skalski (Eds.), Immersed in media: Telepresence in everyday life (pp. 197-228). Routledge.

Lombard, M., \& Ditton, T. (1997). At the heart of it all: The concept of presence. Journal of Computer-Mediated Communication, 3(2), JCMC321. https://doi.org/10.1111/ j.1083-6101.1997.tb00072.x

Lombard, M., \& Jones, M. T. (2015). Defining presence. In M. Lombard, F. Biocca, W. A. Ijsselsteijn, J. Freeman, \& R. Schaevitz (Eds.), Immersed in media: Telepresence theory, measurement, and technology. Springer.

Malle, B. F. (1999). How people explain behavior: A new theoretical framework. Personality and Social Psychology Review, 3(1), 23-48. https://doi.org/10.1207/s15327957pspr0301_2

Martin, D., \& Macrae, C. N. (2007). A face with a cue: Exploring the inevitability of person categorization. European Journal of Social Psychology, 37(5), 806-816. https://doi. org/10.1002/ejsp.445

Martini, M. C., Gonzalez, C. A., \& Wiese, E. (2016). Seeing minds in others-Can agents with robotic appearance have human-like preferences? PloS One, 11(1), e0146310. https://doi.org/10.1371/journal.pone.0146310 
Mayer, R. E. (2014). The Cambridge handbook of multimedia learning. Cambridge University Press.

Moon, Y. (2000). Intimate exchanges: Using computers to elicit self-disclosure from consumers. Journal of Consumer Research, 26(4), 323-339. https://doi.org/10.1086/209566

Morewedge, C. K., Preston, J., \& Wegner, D. M. (2007). Timescale bias in the attribution of mind. Journal of Personality and Social Psychology, 93(1), 1-11.

Mori, M., MacDorman, K. F., \& Kageki, N. (2012). The uncanny valley. IEEE Robotics \& Automation Magazine, 19(2), 98-100.

Mou, Y., Shi, C., Shen, T., \& Xu, K. (2020). A systematic review of the personality of robot: Mapping its conceptualization, operationalization, contextualization and effects. International Journal of Human-Computer Interaction, 36(6), 591-605. https://doi.org/10.10 80/10447318.2019.1663008

Mou, Y., \& Xu, K. (2017). The media inequality: Comparing the initial human-human and human-AI social interactions. Computers in Human Behavior, 72, 432-440. https://doi. org/10.1016/j.chb.2017.02.067

Nass, C. (2004). Etiquette equality: Exhibitions and expectations of computer politeness. Communications of the ACM, 47, 35-37. https://doi.org/10.1145/975817.975841

Nass, C., \& Brave, S. (2005). Wired for speech: How voice activates and advances the human-computer relationship. MIT Press.

Nass, C., Fogg, B. J., \& Moon, Y. (1996). Can computers be teammates? International Journal of Human Computer Studies, 45(6), 669-678. https://doi.org/10.1006/ijhc.1996.0073

Nass, C., \& Lee, K. M. (2001). Does computer-synthesized speech manifest personality? Experimental tests of recognition, similarity-attraction, and consistency-attraction. Journal of Experimental Psychology: Applied, 7(3), 171-181. https://doi.org/10.1037/1076898X.7.3.171

Nass, C., Lombard, M., Henriken, L. \& Steuer, J. (1995). Anthropocentrism and computers. Behaviour and Information Technology, 14(4), 229-238. https://doi. org/10.1080/01449299508914636

Nass, C., \& Moon, Y. (2000). Machines and mindlessness: Social responses to computers. Journal of Social Issues, 56(1), 81-103. https://doi.org/10.1111/0022-4537.00153

Nass, C., Moon, Y., \& Green, N. (1997). Are machines gender neutral? Gender-stereotypic responses to computers with voices. Journal of Applied Social Psychology, 27(10), 864876. https://doi.org/10.1111/j.1559-1816.1997.tb00275.x

Nass, C., \& Steuer, J. (1993). Voices, boxes, and sources of messages: Computers and social actors. Human Communication Research, 19(4), 504-527. https://doi. org/10.1111/j.1468-2958.1993.tb00311.x

Nass, C., Steuer, J., \& Tauber, E. R. (1994). Computer are social actors. Human Factors in Computing Systems, 94, 72-78. https://doi.org/10.1145/259963.260288

Nishio, S., Ishiguro, H., \& Hagita, N. (2007). Geminoid: Teleoperated android of an existing person. In A. C. Philho (Ed.), Humanoid robots: New developments (pp. 343-352). InTech.

Nitto, H., Taniyama, D., \& Inagaki, H. (2017). Social acceptance and impact of robots and artificial intelligence. Nomura Research Institute, 211, 1-15. 
Nomura, T., \& Kanda, T. (2015). Influences of evaluation and gaze from a robot and humans' fear of negative evaluation on their preferences of the robot. International Journal of Social Robotics, 7(2), 155-164. https://doi.org/10.1007/s12369-014-0270-y

Okumura, Y., Kanakogi, Y., Kanda, T., Ishiguro, H., \& Itakura, S. (2013). Can infants use robot gaze for object learning? The effects of verbalization. Interaction Studies, 14(3), 351-365. https://doi.org/10.1075/is.14.3.03oku

Orwig, J. (2014, September 24). More than 7 billion people populate earth and each one has a unique face - Here is why. Business Insider. http://www.businessinsider.com/why-arehuman-faces-so-complex-and-unique-2014-9

Paepcke, S., \& Takayama, L. (2010, March 2-5). Judging a bot by its cover: An experiment on expectation setting for personal robots. In The 2010 th ACM/IEEE International Conference on Human-Robot Interaction (pp. 45-52).

Perry, T. (2014). Suitable technologies opens store staffed only by robots. ISPR. http://ispr. info/2014/12/23/telepresence-robot-store-is-staffed-only-by-robots/

Petronio, S., \& Durham, W. T. (2008). Understanding and applying communication privacy management theory. In D. Braithwaite \& P. Schrodt (Eds.), Engaging theories in interpersonal communication: Multiple perspectives (pp. 309-322). Sage Publications.

Reeves, B., \& Nass, C. (1996). The media equation: How people treat computers, television, and new media like real people and places. CSLI Publications.

Reeves, B., \& Nass, C. (2000). Perceptual bandwith. Communications of the ACM, 43(3), 65-70. https://doi.org/10.1145/330534.330542

Reeves, B., \& Thorson, E. (1986). Watching television: Experiments on the viewing process. Communication Research, 13(3), 343-361. https://doi.org/10.1177/009365086013003004

Reicher, S. D., Spears, R., \& Postmes, T. (1995). A social identity model of deindividuation phenomena. European Review of Social Psychology, 6(1), 161-198. https://doi. org/10.1080/14792779443000049

Rosen, N. O., \& Knäuper, B. (2009). A little uncertainty goes a long way: State and trait differences in uncertainty interact to increase information seeking but also increase worry. Health Communication, 24(3), 228-238. https://doi.org/10.1080/10410230902804125

Rubin, A. M. (1983). Television uses and gratifications: The interactions of viewing patterns and motivations. Journal of Broadcasting, 27(1), 37-51. https://doi. org/10.1080/08838158309386471

Sah, Y. J., \& Peng, W. (2015). Effects of visual and linguistic anthropomorphic cues on social perception, self-awareness, and information disclosure in a health website. Computers in Human Behavior, 45, 392-401. https://doi.org/10.1016/j.chb.2014.12.055

Salem, M., Eyssel, F., Rohlfing, K., Kopp, S., \& Joublin, F. (2013). To err is human(-like): Effects of robot gesture on perceived anthropomorphism and likability. International Journal of Social Robotics, 5(3), 313-323. https://doi.org/10.1007/s12369-013-0196-9

Schmidt, K. L., \& Cohn, J. F. (2001). Human facial expressions as adaptations: Evolutionary questions in facial expression research. Yearbook of Physical Anthropology, 44, 3-24. https://doi.org/10.1002/ajpa.20001

Serrano, J. M., Iglesias, J., \& Loeches, A. (1992). Visual discrimination and recognition of facial expressions of anger, fear, and surprise in 4- to 6-month-old infants. Developmental Psychobiology: The Journal of the International Society for Developmental Psychobiology, 25(6), 411-425. https://doi.org/10.1002/dev.420250603 
Shamekhi, A., Liao, Q. V., Wang, D., Bellamy, R. K., \& Erickson, T. (2018, April). Face value? Exploring the effects of embodiment for a group facilitation agent. In Proceedings of the 2018 CHI Conference on Human Factors in Computing Systems (pp. 1-13). https://doi. org/10.1145/3173574.3173965

Shoemaker, P. J. (1996). Hardwired for news: Using biological and cultural evolution to explain the surveillance function. Journal of Communication, 46(3), 32-47. https://doi. org/10.1111/j.1460-2466.1996.tb01487.x

Short, J., Williams, E., \& Christie, B. (1976). Theoretical approaches to differences between media. In Social Psychology of Telecommunications (pp. 61-66). Wiley.

Slater, M., \& Wilbur, S. (1997). A framework for immersive virtual environments (FIVE): Speculations on the role of presence in virtual environments. Presence: Teleoperators and Virtual Environments, 6, 603-616. https://doi.org/10.1162/pres.1997.6.6.603

Sokol, R. I., \& Strout, S. L. (2007). Understanding human psychology: The integration of social, evolutionary, and cultural studies. Journal of Social, Evolutionary, and Cultural Psychology, 1(1), 1. https://doi.org/10.1037/h0099089

Solomon, J., \& Wash, R. (2014). Human-what interaction? Understanding user source orientation. In Proceedings of the Human Factors and Ergonomics Society Annual Meeting, 58(1), 422-426. https://doi.org/10.1177/1541931214581088

Sproull, L., \& Kiesler, S. (1986). Reducing social context cues: Electronic mail in organizational communication. Management Science, 32(11), 1492-1512. https://doi. org/10.1287/mnsc.32.11.1492

Streater, J., Bockelman Morrow, P., \& Fiore, S. (2012). Making things that understand people: The beginnings of an interdisciplinary approach for engineering computational social intelligence. In The 56th Annual Meeting of the Human Factors and Ergonomics Society (pp. 22-26).

Sundar, S. S., Jia, H., Waddell, F., \& Huang, Y. (2015). Toward a theory of interactive media effects (TIME): Four models for explaining how interface features affect user psychology. In S. S. Sundar (Ed.), The handbook of the psychology of communication technology (pp. 47-86). John Wiley \& Sons, Inc.

Sung, J., Grinter, R. E., \& Christensen, H. I. (2009). "Pimp my Roomba”: Designing for personalization. In Proceedings of the SIGCHI Conference on Human Factors in Computing Systems (pp. 193-196). https://doi.org/10.1145/1518701.1518732

Syrdal, D. S., Dautenhahn, K., Woods, S., Walters, M. L., \& Koay, K. L. (2006, Sept. 6-8). 'Doing the right thing wrong' - Personality and tolerance to uncomfortable robot approaches. In 15th IEEE International Symposium on Robot and Human Interactive Communication (pp. 183-188). IEEE.

Takahashi, K., \& Watanabe, K. (2013). Gaze cueing by pareidolia faces. i-Perception, 4(8), 490-492. https://doi.org/10.1068/i0617sas

Takayama, L., \& Pantofaru, C. (2009, October). Influences on proxemic behaviors in human-robot interaction. In IEEE/RSJ International Conference on Intelligent Robots and Systems (pp. 5495-5502). IEEE.

Tanis, M., \& Postmes, T. (2003). Social cues and impression formation in CMC. Journal of Communication, 53(4), 676-693. https://doi.org/10.1111/j.1460-2466.2003.tb02917.x 
Terzioğlu, Y., Mutlu, B., \& Şahin, E. (2020, March). Designing social cues for collaborative robots: The role of gaze and breathing in human-robot collaboration. In Proceedings of the 2020 ACM/IEEE International Conference on Human-Robot Interaction (pp. 343357). https://doi.org/10.1145/3319502.3374829

Tomasello, M. (2014). The ultra-social animal. European Journal of Social Psychology, 44(3), 187-194. https://doi.org/10.1002/ejsp.2015

Tung, F. W., \& Deng, Y. S. (2007). Increasing social presence of social actors in e-learning environments: Effects of dynamic and static emoticons on children. Displays, 28(4-5), 174-180. https://doi.org/10.1016/j.displa.2007.06.005

Turkle, S. (2012). Alone together: Why we expect more from technology and less from each other. Basic Books.

Vinciarelli, A., Pantic, M., \& Bourlard, H. (2009). Social signal processing: Survey of an emerging domain. Image and Vision Computing, 27(12), 1743-1759. https://doi. org/10.1016/j.imavis.2008.11.007

Vincent, J. (2020, January). Samsung's artificial human project definitely looks like a digital avatar. The Verge. https://www.theverge.com/circuitbreaker/2020/1/5/21050288/ samsung-neon-artificial-human-digital-avatar-project-leaked-video

Waddell, T. F. (2018). A robot wrote this?: How perceived machine authorship affects news credibility. Digital Journalism, 6(2), 236-255. https://doi.org/10.1080/21670811.2017.13 84319

Walters, M. L., Koay, K. L., Syrdal, D. S., Dautenhahn, K., \& Te Boekhorst, R. (2009). Preferences and perceptions of robot appearance and embodiment in human-robot interaction trials. In Proceedings of New Frontiers in Human-Robot Interaction (pp. 136-143).

Walther, J. B. (1996). Computer-mediated communication: Impersonal, interpersonal, and hyperpersonal interaction. Communication Research, 23(1), 3-43. https://doi. org/10.1177/009365096023001001

Walther, J. B., Loh, T., \& Granka, L. (2005). Let me count the ways the interchange of verbal and nonverbal cues in computer-mediated and face-to-face affinity. Journal of Language and Social Psychology, 24(1), 36-65. https://doi.org/10.1177/0261927X04273036

Wang, S. S., Moon, S. I., Kwon, K. H., Evans, C. A., \& Stefanone, M. A. (2010). Face off: Implications of visual cues on initiating friendship on Facebook. Computers in Human Behavior, 26(2), 226-234. https://doi.org/10.1016/j.chb.2009.10.001

Wiltshire, T. J., Snow, S. L., Lobato, E. J. C., \& Fiore, S. M. (2014). Leveraging social judgment theory to examine the relationship between social cues and signals in humanrobot interactions. In Proceedings of the Human Factors and Ergonomics Society Annual Meeting, 58(1), 1336-1340. https://doi.org/10.1177/1541931214581279

Won, A. S., Bailenson, J., Lee, J., \& Lanier, J. (2015). Homuncular flexibility in virtual reality. Journal of Computer-Mediated Communication, 20(3), 241-259. https://doi.org/10.1111/ jcc4.12107

Xu, K. (2019). First encounter with robot Alpha: How individual differences interact with vocal and kinetic cues in users' social responses. New Media and Society, 21(11-12), 2522-2547. https://doi.org/10.1177/1461444819851479

$\mathrm{Xu}, \mathrm{K}$. (2020). Language, modality, and mobile media use experiences: Social responses to smartphone cues in a task-oriented context. Telematics and Informatics, 48, 101344. https://doi.org/10.1016/j.tele.2020.101344 

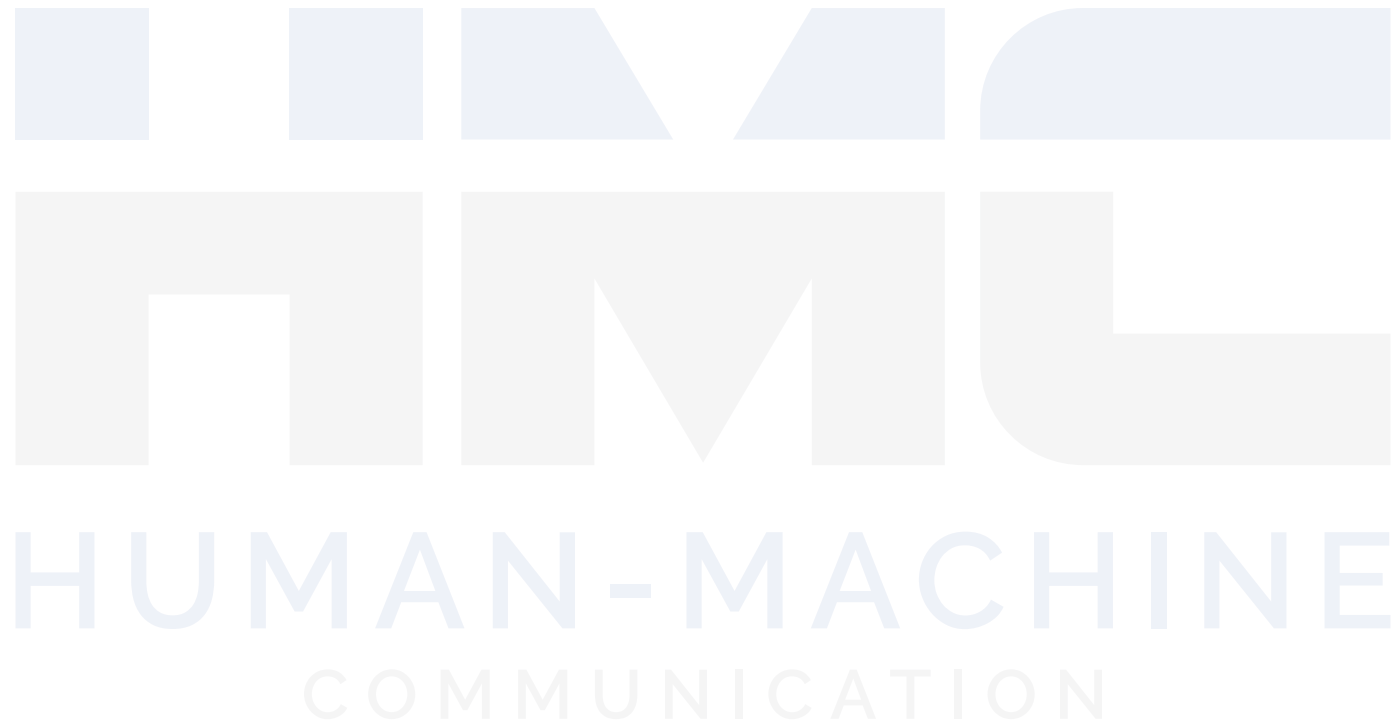